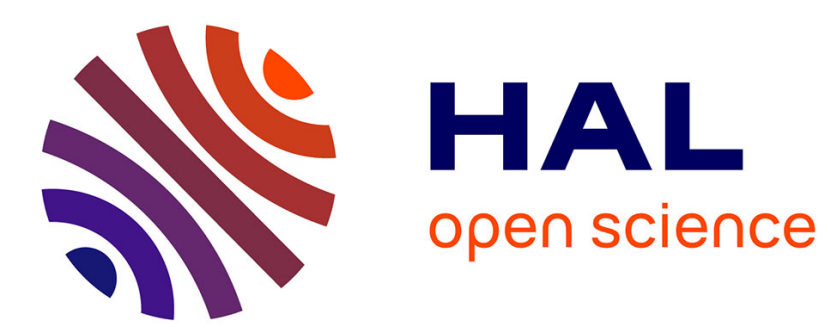

\title{
Les apports d'un observatoire du travail enseignant à un référentiel métier d'enseignants
}

\author{
Audrey Murillo, Marie-Hélène Bouillier-Oudot
}

\section{To cite this version:}

Audrey Murillo, Marie-Hélène Bouillier-Oudot. Les apports d'un observatoire du travail enseignant à un référentiel métier d'enseignants. Travail et apprentissages: revue de didactique professionnelle, 2012. halshs-03087133

\section{HAL Id: halshs-03087133 \\ https://shs.hal.science/halshs-03087133}

Submitted on 7 Jan 2021

HAL is a multi-disciplinary open access archive for the deposit and dissemination of scientific research documents, whether they are published or not. The documents may come from teaching and research institutions in France or abroad, or from public or private research centers.
L'archive ouverte pluridisciplinaire HAL, est destinée au dépôt et à la diffusion de documents scientifiques de niveau recherche, publiés ou non, émanant des établissements d'enseignement et de recherche français ou étrangers, des laboratoires publics ou privés. 


\title{
Les apports d'un observatoire du travail enseignant à un référentiel métier d'enseignants
}

\section{Titre en anglais : Contributions of an observatory of teaching work in a teachers professional system of reference}

\author{
Audrey Murillo, Marie-Hélène Bouillier-Oudot \\ Unité Mixte de Recherche « Education, Formation, Travail, Savoirs » \\ Ecole Nationale de Formation Agronomique \\ Université de Toulouse
}

Mots-clés : travail enseignant, observatoire, referential métier, enseignement agricole

Key words : teaching work, observatory, professional system of reference, agricultural education

\section{Résumé}

Nous proposons dans cette contribution de décrire et questionner le référentiel métier des enseignants de l'enseignement technique agricole français. Pour ce faire, nous nous appuyons sur des résultats issus d'un observatoire du travail enseignant dans l'enseignement agricole. Des résultats sur le temps professionnel et les difficultés perçues par les enseignants nous conduisent à envisager les apports de l'observatoire pour le référentiel métier qui guide la formation des enseignants. Ces apports consistent en une objectivation du contexte de travail des enseignants et de ses liens avec leur activité, la mise en évidence de l'ensemble des situations professionnelles rencontrées par les enseignants ainsi que des situations pouvant être considérées comme critiques. Enfin, nous montrerons les limites de l'observatoire du travail enseignant, et l'intérêt pour la formation d'une approche complémentaire relevant de la didactique professionnelle et analysant l'activité des enseignants dans les situations mises au jour dans le cadre de l'observatoire.

\section{Summary}

We propose in this paper to describe and question the professional system of reference of teachers in French agricultural schools. For this purpose we rely on the results from an 
observatory of teaching work in agricultural schools. Results of the teachers'work schedule and the difficulties they perceive lead us to consider the contributions of the observatory for the professional system of reference which guides teacher training. These contributions consist of an objectification of teachers' working environment and its relationship with their activity, highlighting all professional situations encountered by teachers as well as situations that can be considered critical. Finally, we show the limits of this observatory, and the interest for teachers' training of a complementary approach covered by professional didactics and analyzing the activity of teachers in situations brought to light through the observatory. 
Notre contribution s'inscrit dans la problématique de la professionnalisation de la formation des enseignants qui fait débat depuis une vingtaine d'années (Altet, Paquay, \& Perrenoud, 2006; Develay, 1994; Van Zanten, 2002). Nous proposons une réflexion sur les apports d'un observatoire du travail enseignant et de la didactique professionnelle à la formation à l'enseignement.

La création d'instituts de formation distincts des lieux d'exercice du métier d'enseignant et la formalisation de cursus spécifiques ont rendu nécessaire l'élaboration de références utiles à l'orientation et la mise en œuvre de ces formations. Nous abordons la question des références utiles à la professionnalisation de la formation des enseignants en prenant appui sur une étude conduite actuellement dans le cadre de la mise en place d'un observatoire du travail enseignant au sein de l'enseignement agricole français.

Nous proposons d'analyser dans quelle mesure cette étude est susceptible de renouveler la description du métier formalisée dans le référentiel actuel. Nous mettons également en évidence les apports et les limites de cette démarche, en la situant par rapport à l'étude de l'activité d'enseignement développée par la didactique professionnelle. Nous explorons les complémentarités possibles entre ces deux approches, concernant la production de références utiles à la formation des enseignants.

\section{Problématique}

Notre objet d'étude est la formation des enseignants du Ministère de l'agriculture, de l'alimentation, de la pêche, de la ruralité et de l'aménagement du territoire (MAAPRAT) et les prescriptions dont elle fait l'objet.

Le métier d'enseignant en établissement d'enseignement agricole est décrit dans un référentiel (Ecole nationale de formation agronomique de Toulouse, 2009) élaboré il y a une dizaine d'années par l'institut de formation et validé par le Ministère. Il sert actuellement de cadre de référence pour orienter la formation.

Ce document s'inscrit dans la démarche de référentialisation qui s'est développée depuis les années 1980 dans l'ensemble de l'enseignement professionnel pour répondre au souci de mettre plus directement en correspondance l'offre de formation et la distribution des activités professionnelles (Tanguy, 1994). Dans l'enseignement technique s'est progressivement développée une méthode d'élaboration de ces référentiels selon deux volets :

- une description des activités professionnelles auxquelles prépare le diplôme : le référentiel professionnel, 
- un référentiel de formation, déduit du référentiel professionnel, et qui décline les compétences visées en objectifs de formation.

Pour les organismes de formation, ces référentiels ne constituent qu'un cadre et ne sauraient être assimilés à un programme (Tanguy, 1994, p. 45). Ce sont avant tout des documents d'information permettant d'orienter les choix de l'institution et l'action des formateurs. Ceuxci doivent pouvoir y trouver les éléments permettant :

- de définir les visées de la formation et de construire des parcours de professionnalisation pertinents,

- de définir les contenus de formation aptes à développer les compétences nécessaires pour agir efficacement dans les principales situations professionnelles qui composent le métier.

Ces deux niveaux de références sont complémentaires et leur définition est particulièrement problématique vis-à-vis d'un métier comme celui d'enseignant, régulièrement questionné à la fois sur les missions qu'il doit remplir au sein de la société et sur l'efficacité des pratiques de ceux qui l'exercent. Dans cette contribution, nous analysons le référentiel métier actuel à l'aune d'une étude menée dans le cadre de l'observatoire du travail enseignant dans l'enseignement agricole: quels sont les apports potentiels de cet observatoire pour le référentiel ? Quelles en sont les limites ? En quoi des travaux de didactique professionnelle sur l'activité des enseignants pourraient-ils être complémentaires de l'observatoire pour orienter la formation des enseignants?

\section{Les principales caractéristiques du référentiel métier d'enseignant en établissement d'enseignement agricole}

Le référentiel du métier d'enseignant en établissement d'enseignement agricole (Ecole nationale de formation agronomique de Toulouse, 2009) a été élaboré à l'initiative de l'ENFAT, au moment de rénover le plan de formation des professeurs stagiaires. Validé par le ministère de tutelle, il a constitué une étape importante pour construire la cohérence du plan de formation qui a prévalu jusqu'à la récente réforme dite de «masterisation » modifiant les conditions de recrutement et de formation des enseignants. Ce référentiel reste encore aujourd'hui le seul document de référence pour présenter le métier et le cadre d'orientation de la formation professionnelle. 
Ce document articule au sein d'une matrice quatre grands champs de compétences : la gestion des apprentissages, l'ingénierie, l'analyse de système, les aspects relationnels et éthiques du métier. A chaque champ correspondent trois niveaux d'exercice de l'activité : la classe, la filière de formation et l'établissement en lien avec son territoire d'appartenance. Au sein de cette matrice sont listées les principales tâches afférentes, par exemple :

- au niveau de la gestion des apprentissages à l'échelle de la classe : concevoir et mettre en œuvre des séances d'enseignement, réguler et évaluer des apprentissages...

- au niveau de l'ingénierie à l'échelle de la classe et de la filière de formation : analyser les référentiels et programmes, élaborer une progression, participer à la conception collective de la formation à l'échelle d'une filière, à la conception de projets pédagogiques...

La partie la plus spécifique à l'enseignement agricole porte sur la capacité à conduire une analyse de système. A l'échelle de l'établissement, il s'agit d'analyser son fonctionnement en relation avec son territoire pour être en capacité d'initier et d'organiser des collaborations en interne et des partenariats externes, de contribuer à l'élaboration du projet d'établissement et de développer des projets en lien avec les missions dévolues aux établissements.

Tous les champs de cette matrice participent à parts égales à la description du métier auquel l'institut de formation souhaite préparer le futur enseignant. Peu précis dans la description des tâches composant le métier, le référentiel s'attache principalement à donner du sens à l'une des visées de la formation : la socialisation professionnelle. Il s'agit de préparer les futurs enseignants à entrer dans une institution, l'enseignement agricole, qui a sa culture, une organisation et des missions spécifiques. La fonction d'enseignement dans la classe, reconnue comme étant «le cœur du métier» dans les écrits qui accompagnent ce référentiel, est cependant peu détaillée. Elle est englobée dans un ensemble qui s'attache à valoriser la dimension collective du métier, l'intégration dans un établissement et ses projets. De ce fait, le volet du référentiel relatif à la participation à des missions d'animation et de développement des territoires ruraux qui caractérisent ces établissements est mis en exergue et contribue à l'identification d'un métier propre à ce système éducatif.

Cette analyse fait écho à un ensemble de remarques relatives aux référentiels en général et aux référentiels de métier d'enseignant en particulier. Initiée dans les années 1980 dans la perspective de mieux articuler formation et emploi, la démarche codifiée d'élaboration de référentiels a fait l'objet d'une série d'écrits soulignant l'intérêt et les limites de cette 
démarche (Baradat-Bouillier, 1999; Lenoir, 1993, 2010; Lenoir \& Bouillier-Oudot, 2006; Tanguy, 1994). Dans ces référentiels la description du métier aboutit très souvent à une perte de sens liée au morcellement des tâches prises en compte et à une standardisation de la référence produite. Face à la diversité des contextes et des modes opératoires, quelle «bonne » pratique décrire? En quoi les références produites sont elles représentatives d'un métier? La «normalisation » à laquelle aboutit la démarche tient également au processus social d'élaboration des référentiels, qui met en jeu des rapports de pouvoir entre différentes représentations syndicales n'ayant pas la même vision du métier. La plupart des auteurs s'accordent à dire que l'enjeu principal d'un référentiel professionnel est la construction collective d'une norme sociale décrivant le métier. Ils l'abordent comme construit social traduisant les rapports de force entre les groupes de pression participant à son élaboration. Sa fonction est essentiellement politique.

Concernant le métier d'enseignant, un référentiel présente généralement les orientations prescriptives au regard des missions attribuées au système scolaire (Lenoir, 2010). Prenant en France la forme d'un cahier des charges, il fixe principalement les buts à atteindre, laissant aux enseignants le soin de déterminer les moyens didactiques et pédagogiques à mettre en œuvre pour y parvenir (Rey, 2010, p. 119).

La description du métier d'enseignant en établissement d'enseignement agricole qui sert actuellement de cadre de référence pour la formation présente donc le métier tel que l'institut de formation souhaite le promouvoir. Il a notamment pour fonction de légitimer certaines séquences au sein d'un plan de formation qui fait l'objet de discussions et de tensions entre des formateurs ne partageant une vision unifiée du métier.

Nous allons décrire l'observatoire du travail enseignant, dont les fonctions diffèrent, afin d'envisager les apports de cet observatoire pour la formation des enseignants.

\section{L'observatoire du travail enseignant dans l'enseignement agricole}

\subsection{Genèse et fonctions}

La création en 2010 d'un observatoire du travail enseignant dans l'enseignement technique agricole répond à une préoccupation émergeant des dernières assises consacrées à ce système éducatif. Que sait-on du quotidien du métier d'enseignant aujourd'hui ? Il est banal de dire qu'il s'est considérablement transformé en écho à de nouvelles attentes sociales vis-à-vis de l'école. L'évolution des publics en formation et des modes de management d'établissements porteurs de marges d'autonomie plus importantes ont transformé le travail des enseignants ces 
dernières décennies. Au-delà de ces considérations générales, le souci formulé par les participants à ces assises était également de faire un point sur les pratiques enseignantes et les spécificités de ce système éducatif, comme la pluridisciplinarité et les liens avec les territoires ruraux, qui font parfois dire qu'il existerait un métier d'enseignant spécifique au système agricole.

L'étude menant à la création d'un observatoire est donc commanditée par le ministère chargé de l'agriculture, qui assure la tutelle à la fois de l'ensemble des établissements d'enseignement agricole et de l'institut ayant en charge la formation des enseignants affectés dans ces établissements. Ceux-ci, qualifiés d'Etablissements public locaux d'enseignement et de formation professionnelle agricole (EPLEFPA), présentent un ensemble de caractéristiques propres. Les formations proposées recouvrent des filières scientifiques, technologiques et professionnelles en formation initiale par voie scolaire mais également des formations par apprentissage et des formations professionnelles pour adultes. Chaque EPLEFPA regroupe ainsi, autour d'une exploitation agricole ou d'un atelier technologique à vocation pédagogique, un ou plusieurs lycée(s) agricole(s), un ou plusieurs Centre(s) de formation d'apprentis (CFA) et Centre(s) de formation professionnelle et de promotion agricoles (CFPPA).

Tous ces centres appartiennent à la même entité juridique et administrative : l'EPLEFPA, lieu d'affectation des enseignants qui ont ainsi la possibilité d'intervenir auprès de publics différents, remplissant conjointement les fonctions de professeurs et de formateurs d'adultes ou d'apprentis. C'est l'ensemble de ces fonctions qu'il nous est demandé de décrire sous le vocable de travail enseignant dans l'enseignement agricole.

Les objectifs de l'étude sont fixés en ces termes dans un courrier du ministère : «L'ambition est de se donner les moyens de décrire la réalité constitutive du travail enseignant par le biais d'une grille de lecture elle-même adossée à des mesures précises à la fois qualitatives et quantitatives. Ce dispositif a pour vocation de s'inscrire dans la durée et à produire des états des lieux réguliers fondés sur la collecte, l'analyse et la mise en perspective des informations recueillies ».

L'étude est confiée à l'équipe de recherche en éducation de l'institut de formation des enseignants de lycées agricoles ${ }^{1}$. L'une de ses retombées sera d'éclairer les orientations de la formation initiale et continue des enseignants de ce système. C'est dans cette perspective que

\footnotetext{
${ }^{1}$ Unité Mixte de Recherche « Education Formation Travail et Savoirs » (EFTS) dont sont membres les deux auteures en poste à l'Ecole nationale de formation agronomique de Toulouse (ENFAT).
} 
nous présentons ici le cadre théorique et méthodologique, puis une partie des résultats obtenus.

\subsection{Cadre théorique et méthodologique}

Pour circonscrire et préciser la notion de « travail enseignant » qui constitue le point de départ de cette étude, nous nous sommes appuyés sur l'analyse qu'en font Tardif et Lessard (1999) à partir de trois dimensions issues des travaux de De Coster et Pichault (1998) :

- le travail comme activité qui regroupe « les structures organisationnelles dans lesquelles se déroule cette activité, structures qui la conditionnent de multiples façons » et « le déroulement de cette activité, c'est-à-dire les interactions continuelles, au sein du processus de travail concret, entre le travailleur, son produit, ses buts, ses outils, ses savoirs et les résultats du travail »,

- le travail comme expérience, c'est-à-dire « le travail tel qu'il est vécu et signifié par et pour lui-même ». Cette expérience peut être entendue selon deux sens : soit comme un «processus d'apprentissage spontané qui permet au travailleur d'acquérir des certitudes quant à la façon de contrôler les faits et les situations de travail qui se répètent ", soit comme un processus fondé sur « l'intensité et la signification d'une situation vécue par un individu».

- le travail comme statut qui renvoie à la question de « l'identité du travailleur à la fois dans l'organisation du travail et dans l'organisation sociale, dans la mesure où celles-ci fonctionnent selon une imposition de normes et de règles qui définissent les rôles et les positions des acteurs ».

Ces trois dimensions ont été déclinées en 15 facettes du travail enseignant, présentées dans le tableau ci-après. En gras sont mentionnés les aspects sur lesquels nous porterons notre attention dans cet article.

Tableau 1. Opérationnalisation des dimensions et facettes du travail enseignant

\begin{tabular}{|c|c|c|}
\hline $\begin{array}{c}\text { Dimensions du } \\
\text { travail }\end{array}$ & Facettes & Opérationnalisation des facettes \\
\hline \multirow{3}{*}{$\begin{array}{c}\text { Le travail comme } \\
\text { activité }\end{array}$} & $\begin{array}{c}\text { Le cadre organisationnel } \\
\text { du travail }\end{array}$ & $\begin{array}{c}\text { Informations sur les établissements, le pilotage } \\
\text { par l'équipe de direction, la perception du } \\
\text { pilotage et de l'organisation }\end{array}$ \\
\cline { 2 - 3 } & $\begin{array}{c}\text { Les fonctions spécifiques } \\
\text { au sein de l'établissement }\end{array}$ & Professeur principal, coordonnateur de filière \\
\cline { 2 - 3 } & \multicolumn{2}{|c}{} \\
\hline
\end{tabular}




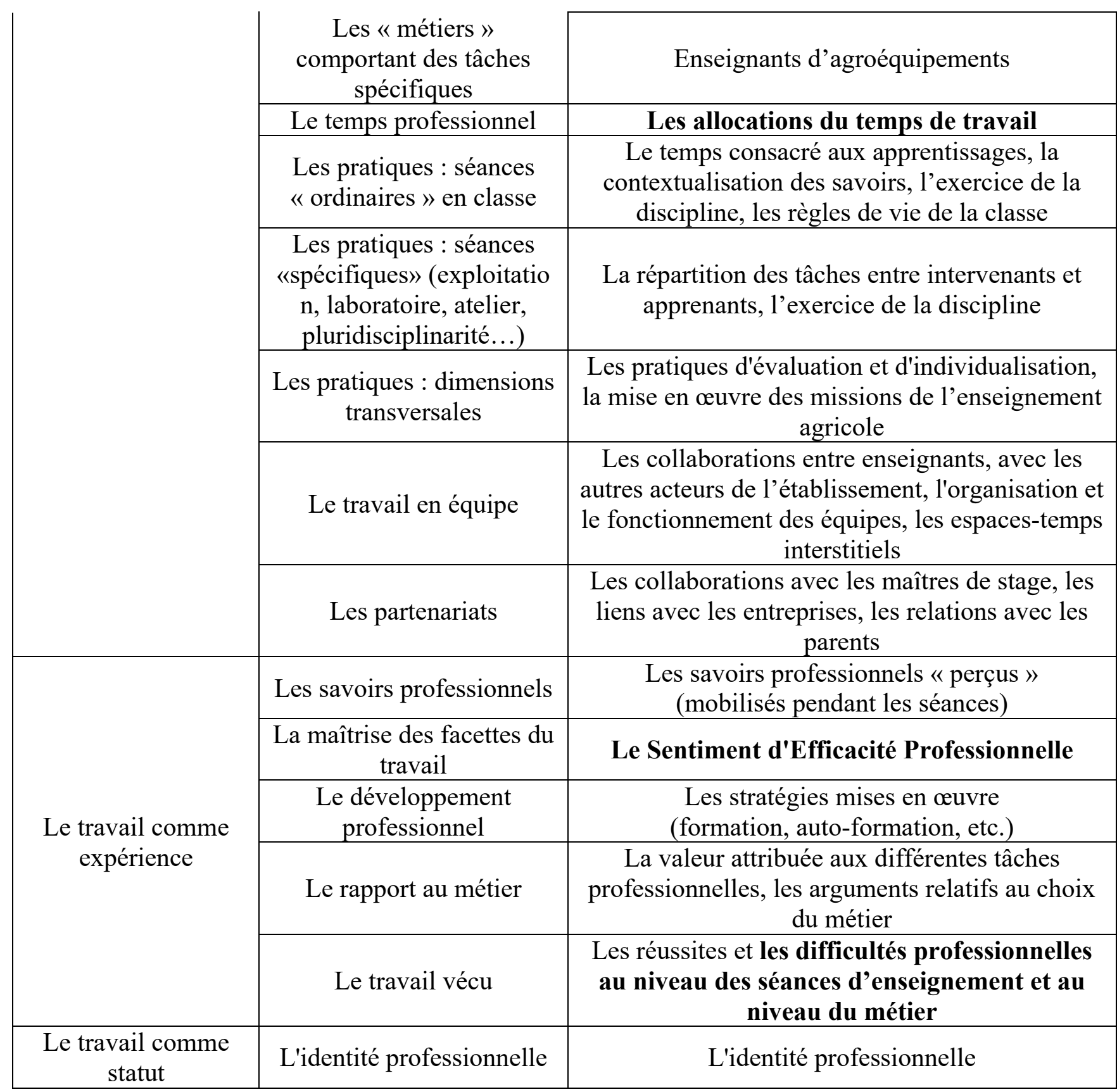

Notons que les facettes présentées dans ce tableau ne se limitent pas aux pratiques d'enseignement, « qui mettent en jeu un enseignant et un groupe d'élèves au sein d'une situation pédagogique », mais touchent plus largement aux pratiques enseignantes, « ensemble des pratiques professionnelles de l'enseignant » (Marcel, 2004, p. 14) et à leur contexte. Dans l'enseignement agricole, ce champ des pratiques professionnelles intègre la participation à des missions dévolues par la loi aux établissements qui, au-delà de l'enseignement, de la formation continue, de l'insertion sociale et professionnelle des jeunes, doivent assurer des fonctions d'animation et de développement du milieu rural et d'expérimentation en lien avec la recherche agricole. 
En 2010-2011, une première phase de l'étude ${ }^{2}$ a consisté à tester dans trois établissements publics locaux un protocole de recueil de données s'appuyant sur des observations, entretiens et questionnaires (Marcel, Murillo, \& Bouillier-Oudot, 2011). En 2011-2012, une deuxième phase a permis de produire des connaissances sur le travail des enseignants, à partir d'une enquête s'étant déroulée dans dix autres établissements d'enseignement agricole.

Les données recueillies portent sur des pratiques à la fois déclarées et observées ; les pratiques observées n'étant pas inférables des pratiques déclarées (Maurice, 2006; Trinquier, 2010), il est nécessaire de réaliser des observations des séances d'enseignement.

Pour ne pas dissocier l'observation des pratiques des structures organisationnelles dans lesquelles elles prennent sens, une équipe pédagogique de chaque centre (lycée, CFA et/ou CFPPA) a été investiguée dans chacun des dix établissements, soit trente équipes au total. 192 enseignants ${ }^{3}$ ont répondu à nos questionnaires; nous avons assisté à 74 séances et réalisé autant d'entretiens avec les enseignants observés.

\subsection{Méthode et premiers résultats}

Nous n'exposons ici qu'une partie des résultats obtenus. Ceux-ci sont mobilisés dans ce texte comme illustration au service d'une démonstration des liens possibles entre les données issues de l'observatoire et l'orientation de la formation initiale des enseignants.

Nous avons choisi de mettre en avant deux séries de données : l'allocation du temps professionnel et les difficultés que les enseignants déclarent rencontrer, en lien direct avec la problématique qui nous préoccupe ici : construire des références utiles à la formation des enseignants.

\subsubsection{L'allocation du temps professionnel}

\section{a) Méthode}

Nous avons estimé le temps consacré par les enseignants à différents types d'activités professionnelles, que ces activités soient fortement repérées et institutionnalisées (par exemple: heures de cours, réunions...) ou réalisées dans des lieux et à des moments échappant à toute visibilité (par exemple : préparation des cours, travail administratif...).

\footnotetext{
2 Nous tenons à remercier Jean-François Marcel, Laurent Fauré, Julie Blanc et Brigitte Laquièze, qui ont participé à la conception de cette étude et/ou au recueil des données présentées dans cette communication.

${ }^{3}$ Par le terme «enseignant», nous englobons à la fois les «professeurs» (exerçant dans les lycées) et les « formateurs » (exerçant dans les CFA et CFPPA).
} 
Nous ne nous situons donc pas dans une approche microscopique du temps d'enseignement voire d'apprentissage, qui permettrait de prendre en compte différents moments infra-séance, par exemple à l'instar de Fisher, Berliner, Filby et al. (1978) ou Bressoux, Bru, Altet et Leconte-Lambert (1999). Notre approche est plus mésoscopique, et distingue sur l'échelle d'une semaine les activités d'enseignement, de préparation, de correction, etc.

Méthodologiquement, il nous a paru très délicat de demander aux enseignants de compléter précisément un emploi du temps au fur et à mesure de leurs activités (auto-observation), ou de les observer nous-mêmes sur de longues durées et en plusieurs lieux. La technique choisie a donc consisté à demander aux enseignants de renseigner, sur un fichier informatisé, leur emploi du temps de la semaine passée, à l'aide de leur agenda. L'emploi du temps d'une semaine n'étant pas nécessairement représentatif des autres semaines de l'année, nous avons demandé aux enseignants d'indiquer l'allocation de leur temps de travail pour deux autres semaines contrastées. Pour cela, les enseignants se sont basés sur des calculs automatiques réalisés sur fichier informatique à partir de l'emploi du temps de la semaine précédente ${ }^{4}$.

\section{b) Résultats}

Les résultats présentés ici ne prennent pas en compte les emplois du temps des enseignants à temps partiel; ils sont basés sur les emplois du temps de 86 enseignants à temps plein exerçant dans dix établissements. Sur une semaine déterminée (la semaine précédant le recueil de données), les enseignants disent travailler en moyenne 41 heures et 32 minutes (hors durées consacrées aux trajets).

Sur ces 41 heures, seules 18, soit moins de la moitié, sont consacrées aux enseignements à proprement parler (heures en présence des apprenants). Notons toutefois une importante dispersion : un enseignant a déclaré avoir enseigné seulement 2 heures, un autre 30 heures. Rappelons que ces enseignants sont à temps plein (soit 18 heures d'enseignement prévues, avec éventuellement des heures supplémentaires). Les variations observées sont le signe d'une répartition inégale de la charge de cours sur l'année scolaire ; par exemple, en Centre de formation d'apprentis, des variations sont liées au fait que les apprentis passent des semaines entières en entreprise, et d'autres dans l'établissement.

\footnotetext{
${ }^{4}$ Par exemple, il est calculé pour un enseignant que le temps de préparation de cours de la semaine précédente est égal à 7 heures. L'enseignant peut se baser sur cette durée pour estimer le temps de préparation lors d'une autre semaine de l'année. Ceci permet de prendre pour point de départ une durée cumulée calculée à partir d'indications plus précises, et non une durée cumulée estimée directement par l'enseignant (durée qui risquerait d'être assez éloignée de la réalité).
} 
La préparation des cours et la correction représentent en moyenne $27 \%$ du temps de travail. Le quart de temps restant est consacré à diverses activités : réunions, travail administratif, activités relatives aux fonctions et missions spécifiques (coordonnateur de filière, chargé de mission...), participations à des jurys, rencontres avec des parents, avec des maîtres de stage, etc. De grandes disparités selon les enseignants sont également à noter : certains disent, lors d'une semaine donnée, n'avoir jamais travaillé au contact d'autres personnes que les élèves, tandis que d'autres déclarent avoir consacré plus de 7 heures à ces activités avec des collègues, le conseiller principal d'éducation, la direction, les parents d'élèves, des professionnels, etc.

Concernant les lieux de travail, en moyenne, presque 10 heures sont passées à travailler hors établissement (notamment à domicile).

Enfin, notons que 58\% des enseignants interrogés exercent des fonctions ou missions spécifiques: coordonnateurs de filières, professeurs principaux, chargés de coopération internationale... Ces activités donnent parfois lieu à des décharges. Dans tous les cas, l'importante proportion d'enseignants concernés montre que même si elles ne sont pas toujours au cœur du métier, ces activités risquent fort d'être exercées par tout enseignant à un moment de sa carrière. Or, des entretiens avec les enseignants indiquent le manque de formation et l'utilité de l'aide des collègues pour assumer ces missions ${ }^{5}$, ainsi que la charge de travail importante, conduisant certains enseignants à moins s'investir dans les activités d'enseignement $^{6}:$ le temps disponible n'est pas sans lien avec les difficultés perçues.

\subsubsection{Les difficultés professionnelles perçues par les enseignants}

a) Méthode

Nous approchons les difficultés perçues par les enseignants de diverses manières :

- par des difficultés très contextualisées, relativement à des séances venant d'être menées par un enseignant. 74 séances ont été observées : 34 séances «ordinaires » et 40 séances « spécifiques» (travaux pratiques, laboratoire, atelier, pluridisciplinarité...). Lors d'un entretien suivant immédiatement chaque séance, l'enseignant évoque «à chaud» les difficultés éventuellement rencontrées. S'ensuit une question qui demande une prise de distance plus importante de la part de l'enseignant: nous l'interrogeons sur les difficultés

\footnotetext{
${ }^{5}$ Extraits d'entretiens exploratoires : «C'était ma première année d'exercice, le diplôme venait d'être créé, je me suis appuyé sur les autres coordo.» « On nous largue coordo sans aucune formation préalable... Heureusement le directeur a été coordo, si j'ai une difficulté je vais le voir. Heureusement on s'entend bien. »

${ }^{6}$ Extraits d'entretiens exploratoires : «Je ne compte pas mon temps, c'est bien plus que la décharge officielle. » «Ca use sur la durée, il y a conflit entre le temps de coordination et le temps que je prends pour préparer mes cours. » « J'ai des cours où j'ai besoin de faire de la veille, c'est difficile. »
} 
qu'il a surmontées avec l'expérience, mais qu'il aurait sûrement rencontrées, débutant, lors d'une séance similaire.

- par des difficultés générales, non centrées sur un moment particulier. Un questionnaire a été conçu suite à des entretiens exploratoires. Les difficultés peuvent être relatives aux situations d'enseignement, mais aussi à d'autres situations professionnelles (réunions, échanges avec la direction...). 163 enseignants ont complété cette section du questionnaire.

- par le Sentiment d'Efficacité Professionnelle (Bandura, 2003; Marcel, 2009). D'après une liste de 29 items mentionnant des activités professionnelles, nous avons demandé à chaque enseignant, par questionnaire, dans quelle mesure il estimait maitriser chacune de ces activités. Nous obtenons ainsi une mesure de son Sentiment d'Efficacité Professionnelle. 178 enseignants ont complété cette section du questionnaire.

\section{b) Résultats}

Relativement aux 74 séances d'enseignement observées, les difficultés citées par les enseignants se répartissent ainsi $^{7}$.

\footnotetext{
${ }^{7}$ Chaque enseignant pouvait mentionner une ou plusieurs difficultés : 98 difficultés ont été évoquées par les 74 enseignants interrogés.
} 


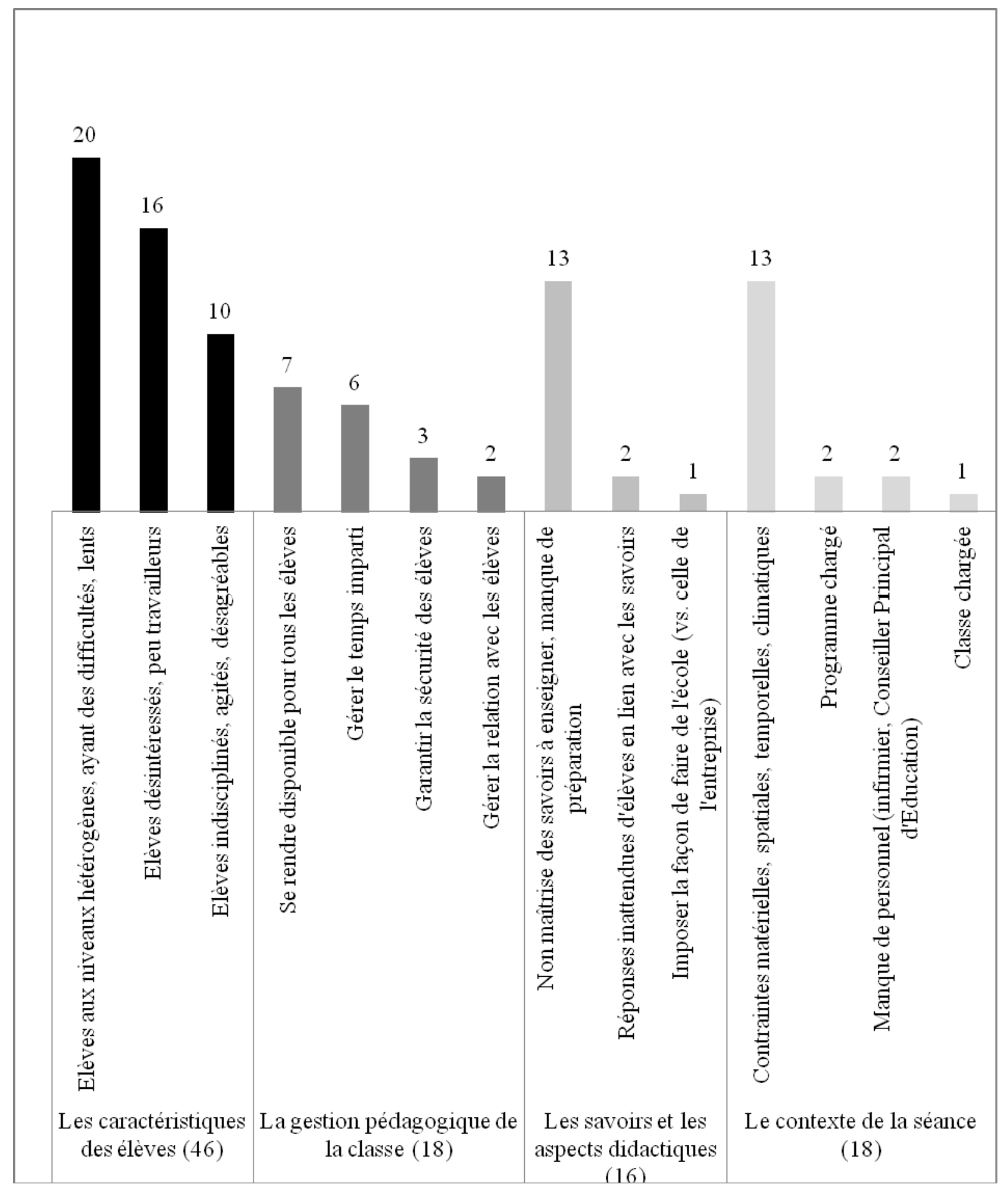

Schéma 1 - Difficultés mentionnées par les enseignants (nombre d'occurrences), concernant les séances

De précédents travaux mettent également en évidence ces difficultés : les enseignants sont à la fois préoccupés par la « gestion de la classe » et la « gestion de la matière » (Goigoux, 2006; Veyrunes, Gal-Petitfaux, \& Durand, 2007). La gestion de l'hétérogénéité de la classe est une difficulté majeure (Murillo, 2010; Wanlin \& Crahay, 2009). Les difficultés perçues comme 
surmontées avec l'expérience mais existant en début de carrière sont présentées dans le graphe ci-après.

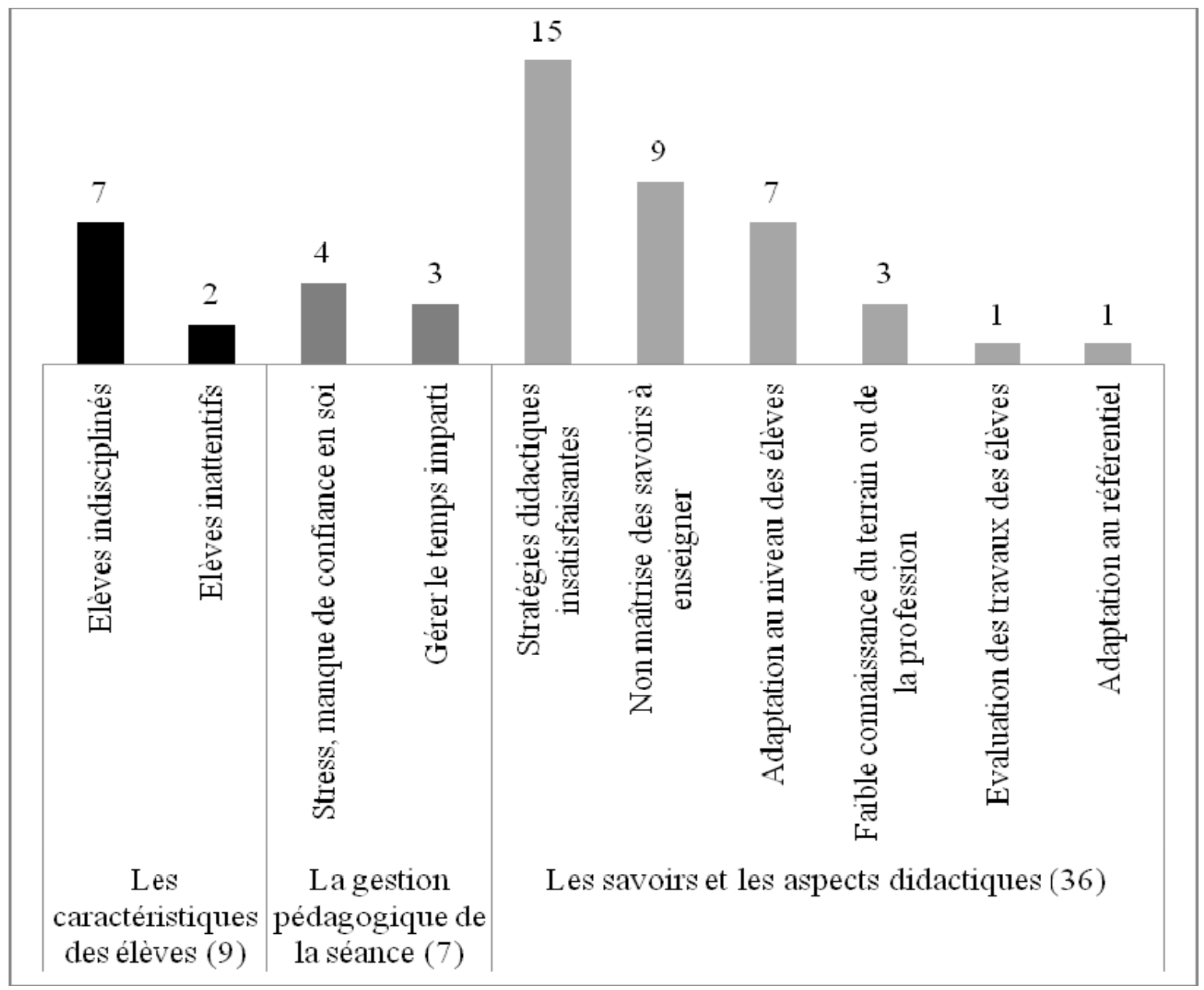

\section{Schéma 2 - Difficultés résolues mentionnées par les enseignants (nombre d'occurrences)}

Si nous mettons en regard les difficultés rencontrées durant la séance qui vient de se dérouler et celles en début de carrière, nous constatons que :

- le contexte passé n'est plus évoqué (en effet, cette partie de l'entretien est décontextualisée) ;

- les problèmes relatifs aux savoirs et aux aspects didactiques étaient bien plus présents en début de carrière, et sont en grande partie considérés comme résolus. En revanche, la prise en compte des caractéristiques des élèves et la relation pédagogique de la séance focalisent toutes les difficultés. 
163 enseignants se sont prononcés, par questionnaire ${ }^{8}$, sur les difficultés relatives au métier (de façon générale). Pour chaque difficulté potentielle, nous indiquons le pourcentage d'enseignants considérant cette difficulté comme « très importante » ou « assez importante ».

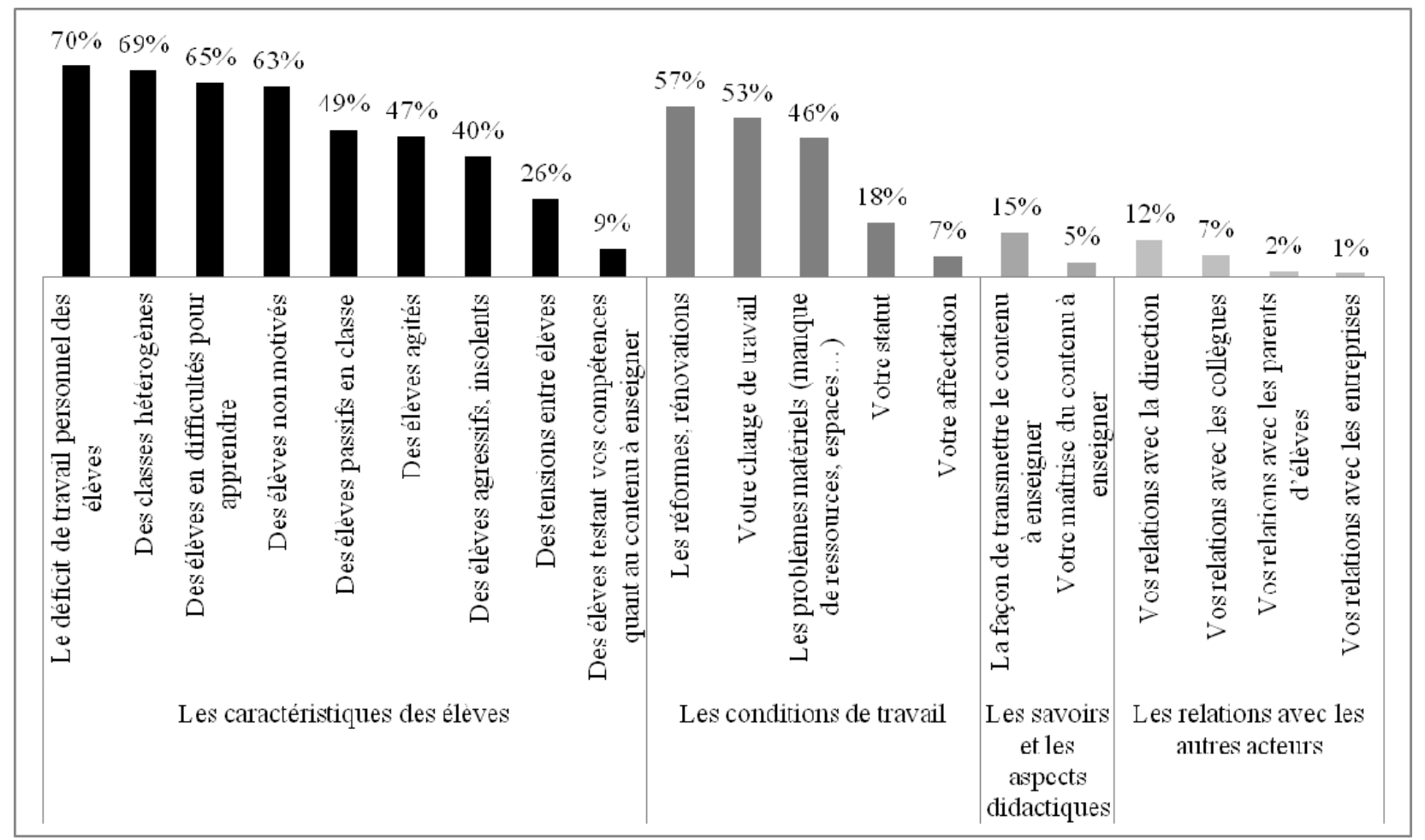

\section{Schéma 3 - Difficultés relatives au métier mentionnées par les enseignants (nombre d'occurrences)}

Nous retrouvons le même type de difficultés au niveau du métier qu'au niveau de la séance : la gestion de l'élève supplante les difficultés relatives aspects didactiques, aux conditions de travail et aux relations aux collègues et partenaires.

Notons de grandes différences entre la formation d'adultes (CFPPA) où les difficultés se cristallisent dans le statut (beaucoup d'enseignants ne sont pas titulaires), et les lycées et CFA où les difficultés relèvent davantage des élèves et des relations avec les autres acteurs.

Enfin, la mesure des Sentiments d'Efficacité Professionnelle des enseignants montre que sur les 29 items listés, 10 posent particulièrement des difficultés : plus de $20 \%$ des enseignants (de $21 \%$ à $34 \%$ selon l'item) disent rencontrer «quelques difficultés » ou « beaucoup de

\footnotetext{
${ }^{8}$ Le questionnaire a été conçu après l'analyse de 13 entretiens exploratoires.
} 
difficultés » au sujet de ces items. Ces 10 items (que les enseignants disent maîtriser le moins) sont, par ordre de difficulté décroissante :

- l'utilisation de supports audiovisuels, informatique,

- l'anticipation du niveau et des difficultés des élèves,

- la gestion des comportements d'indiscipline,

- l'anticipation du temps nécessaire,

- la mise en œuvre de séances pluridisciplinaires,

- la participation à des instances de l'établissement (conseil d'administration...),

- les échanges avec les partenaires extérieurs associatifs et culturels,

- les collaborations avec le directeur d'exploitation et du hall technologique,

- la déclinaison des référentiels sur l'année scolaire,

- les échanges avec les partenaires extérieurs institutionnels (élus, chambre d'agriculture...).

Après l'utilisation des Technologies de l'Information et de la Communication, nous retrouvons dans les difficultés des enseignants la focalisation sur les élèves (qu'il s'agisse de leurs comportements d'indiscipline ou de leur niveau) ainsi que la gestion du temps. Certaines prescriptions sont également difficilement maîtrisées selon les enseignants (prise en compte des référentiels, mise en place de séances pluridisciplinaires), tout comme le travail avec des acteurs au sein de l'établissement et hors établissement.

Finalement, les difficultés des enseignants relèvent de situations qui sont loin de se limiter aux situations d'enseignement à proprement parler.

\section{Quels apports de l'observatoire vis-à-vis du référentiel métier d'enseignant en établissement d'enseignement agricole?}

Nous identifions trois champs concernant les apports de l'observatoire du travail enseignant vis-à-vis du référentiel métier.

\subsection{La mise au jour des situations qui composent effectivement le métier}

Face aux discours chargés d'idéologie portant sur le métier d'enseignant, les données issues de l'observatoire constitueront pour l'ensemble de la communauté une référence plus objective du métier. Cette fois, ce sont les situations professionnelles du terrain qui seront 
exposées, et non pas celles que l'on souhaiterait y trouver. C'est un enjeu majeur de l'observatoire, qui est chargé de produire des données précises quantitatives et qualitatives. Cette objectivité est certes relative, car la photographie produite est soumise à des « effets de cadrage ", c'est-à-dire à des choix théoriques et méthodologiques qui conditionnent la construction des données. Toutefois, cette référence permet de mettre en évidence les pans de l'activité qui ne sont pas pris en charge par la formation, ceux qui sont génériques du métier et ceux qui relèvent davantage d'une organisation locale.

Par exemple, les données présentées ci-dessus montrent que ce qui est défini comme le cœur du métier, c'est-à-dire le face à face avec les élèves, représente moins de la moitié du temps de travail des enseignants. Un grand nombre d'entre eux assure des responsabilités et des fonctions complémentaires, et estime qu'ils ne sont pas assez préparés à ces fonctions. L'observatoire pourrait ainsi contribuer à une redistribution des objectifs assignés à la formation initiale des enseignants.

\subsection{La contribution à une meilleure connaissance des contextes de travail des enseignants dans les EPLEFPA}

Le référentiel produit une vision du métier décontextualisée. L'un des choix méthodologiques importants qui fonde l'observatoire est de prendre comme base d'enquête les structures selon lesquelles est organisé le fonctionnement pédagogique de l'établissement. Ainsi une filière de formation est choisie dans chaque centre constitutif et tous les enseignants de cette filière sont enquêtés. Les modes d'organisation et de pilotage sont décrits pour chaque établissement et chaque centre constitutif, chaque filière (mode de coordination au sein de l'équipe).

L'ambition de l'observatoire est de décrire de manière fine et rigoureuse ce que nous avons appelé « la sphère intrinsèque » du travail enseignant, c'est-à-dire celle qui relève de la marge d'autonomie de l'enseignant qui est composée à la fois des différentes activités professionnelles, du travail partagé et du travail signifié. Elle est en lien avec des variables relevant de ce que nous avons appelé « la sphère extrinsèque » du travail enseignant. Cette sphère qui échappe à l'initiative de l'enseignant est composée de l'organisation du travail et des différents niveaux de pilotage au sein de l'établissement, du contexte institutionnel, des profils des publics accueillis et des caractéristiques socioprofessionnelles des enseignants. Les descriptions fournies par l'observatoire et l'analyse de ces relations contribuent à mieux caractériser le travail enseignant à destination de leurs formateurs, qui pourront s'en saisir dans la construction des situations de formation proposées. La demande récurrente d'une formation plus concrète de la part des futurs enseignants provient largement du décalage entre 
les références mobilisées en centre de formation et les situations au sein desquelles ils ont à prendre des décisions durant leurs stages. Cette objectivation des conditions de travail et des liens entre pratiques et organisations pourrait contribuer à favoriser le dialogue entre les formateurs, les professeurs stagiaires et leurs conseillers pédagogiques.

\subsection{La mise en évidence des situations critiques rencontrées par les enseignants}

C'est certainement au niveau de la mise en évidence des difficultés rencontrées que l'expression des enseignants enquêtés est la plus chargée de sens pour la formation.

Au-delà de la description «froide » des situations de travail, une hiérarchisation basée non plus sur leur fréquence, mais sur les difficultés rencontrées fournit un autre type d'information sur le métier et les besoins de formation. On constate que l'utilisation des nouvelles technologies, la gestion des perturbations des élèves, la prise en charge de leur hétérogénéité et de leurs difficultés représentent les dimensions que les enseignants ont le sentiment de moins maîtriser, même lorsqu'ils sont expérimentés. D'autres écrits montrent que ce sont sur ces aspects du métier que les enseignants sont démunis et vis-à-vis desquels ils attendent un appui de la formation.

On touche là encore à une limite de la démarche de référentialisation et à une caractéristique des prescriptions propres au travail enseignant, qui laissent une marge d'autonomie importante aux acteurs (Leblanc, Ria, Dieumegard, Serres, \& Durand, 2008). Ces situations d'interaction comportent une part d'improvisation et d'adaptation au contexte importantes et se prêtent mal à une description codifiée et normative.

\section{Les limites de l'observatoire et l'intérêt pour la formation d'une approche complémentaire relevant de la didactique professionnelle}

L'observatoire propose une description des principales situations de travail représentatives du métier tel qu'il s'exerce en établissement d'enseignement agricole. Il contribue également à pointer les situations qui posent le plus de problèmes aux enseignants actuellement. Une approche qualitative, portant sur un nombre d'enseignants plus restreint mais s'intéressant à la dynamique de leur activité, pourra compléter l'étude initiale. Les apports potentiels de la didactique professionnelle à la formation se situent, selon nous, dans la possibilité de produire de nouvelles références sur les concepts schèmes des enseignants à partir d'une analyse de leur activité en situation professionnelle. Il s'agit d'analyser la façon dont les enseignants en situation orientent leur action dans un certain nombre de tâches essentielles du métier. La mise au jour des schèmes développés par des praticiens expérimentés permettrait de combler 
l'hiatus reconnu entre les savoirs enseignés en formation d'enseignant et les savoir-faire développés par les praticiens en situation. En effet, si le couple "schème-situation » est organisateur, l'observatoire a permis de repérer les situations significatives et critiques, mais pas les schèmes associés.

Trois exemples issus des résultats de l'observatoire nous permettront de développer nos propos :

- Les enseignants indiquent qu'une de leurs difficultés est l'utilisation de TICE. Dans ce cas, il est possible de s'appuyer sur des travaux (Guin \& Trouche, 1999; Numa Bocage, Clauzard, \& Monchaux, 2011) portant sur l'appropriation d'artefacts technologiques par les enseignants. Une part de l'activité associée à ces artefacts est aisément observable, et les critères de réussite de l'utilisation des artefacts sont en partie objectivables et associés à une courte échelle de temps (exploitation d'une partie de l'artefact par l'enseignant, les élèves, utilisation de la procédure la plus rapide...). Même s'il ne s'agit pas pour l'enseignant d'appliquer uniquement des procédures, maîtriser ces dernières peut assez facilement faire l'objet d'une formation.

- Une autre difficulté indiquée par les enseignants est la gestion des difficultés des élèves en classe. Rogalski (2003) a montré que les enseignants expérimentés, contrairement aux novices, pouvaient repérer non seulement les difficultés des élèves, mais également les situer dans une dynamique d'apprentissage et proposer des solutions de remédiation. Les résultats de l'observatoire semblent montrer que tous les enseignants n'ont pas construit ces savoir-faire. Une étude sur les prises d'information par les enseignants qui ne rencontrent pas ces difficultés pourrait compléter l'observatoire du travail enseignant. Toutefois, les critères de réussite sont en partie subjectifs, mais surtout difficilement observables, puisque référant à des échelles de temps différentes : court terme lors de la séance, moyen terme lors de la séquence, long terme lors de l'année (Rogalski, 2003; Veyrac \& Bouillier-Oudot, 2011).

- Enfin, les enseignants mentionnent également l'indiscipline des élèves dans les difficultés rencontrés. De précédents travaux (Goigoux, 2006) ont pu montrer que la gestion de la classe était étroitement liée à la gestion de la matière. Comment certains enseignants, en maîtrisant les aspects didactiques, parviennent-ils à centrer l'activité des élèves sur les savoirs en jeu ? Les réponses à ces questions pourraient compléter l'observatoire du travail enseignant et apporter des éléments pour la formation, éventuellement adaptés aux spécificités de l'enseignement agricole. 


\section{Conclusion}

Nous conclurons en revenant sur les deux termes de notre problématique :

- construire des références utiles à la formation des enseignants,

- définir et mettre en œuvre une formation permettant aux jeunes enseignants de se situer dans cette profession au sein d'une institution, l'enseignement agricole, et d'agir efficacement dans les principales tâches qui composent le métier.

Définir une formation recouvre à la fois une visée politique (quel professionnel veut-on former pour quelle école ?) et une visée pragmatique (quels contenus de formation proposer pour former des enseignants compétents ?).

L'observatoire du travail enseignant apporte des éléments pour une visée pragmatique, en contribuant à construire une description à la fois globale et précise du métier d'enseignant à partir des données scientifiques et représentatives de la profession. Ces données devraient constituer une référence forte pour alimenter les choix d'orientation de la formation : définir les objectifs de formation et hiérarchiser les priorités. Ce type de référence peut permettre de construire des outils d'accompagnement des parcours de professionnalisation, comme des portfolios.

La mise en évidence des situations de travail les plus représentatives et les plus critiques du métier constitue un apport important et pose un cadre de références pour d'autres démarches complémentaires, comme celles mises en œuvre par la didactique professionnelle. Mayen, Métral et Tourmen (2010) proposent une nouvelle approche des référentiels fondée sur une description et une caractérisation des situations de travail avec lesquelles les professionnels ont ou auront à « se débrouiller ». L'un des objectifs du travail d'élaboration d'un référentiel de situations est de mettre au jour les éléments invariants d'une classe de situations donnée et sa structure conceptuelle (Pastré, Mayen et Vergnaud, 2006), c'est-à-dire l'ensemble des concepts organisant l'action et servant à la guider chez les sujets ayant une activité efficace, et pouvant être inclus dans une ingénierie de formation.

\section{Bibliographie}

Altet, M., Paquay, L., \& Perrenoud, P. (2006). Former des enseignants professionnels : quelles stratégies? Quelles compétences? Bruxelles: De Boeck.

Bandura, A. (2003). Auto-efficacité. Le sentiment d'efficacité personnelle. Bruxelles: De Boeck.

Baradat-Bouillier, M.-H. (1999). L'introduction des problématiques environnementales dans l'enseignement agricole français. Thèse de doctorat. Thèse de doctorat, Université des Sciences Sociales de Toulouse I, Toulouse. 
Bressoux, P., Bru, M., Altet, M., \& Leconte-Lambert, C. (1999). Diversité des pratiques d'enseignement à l'école élémentaire. Revue Française de Pédagogie, 126, 97-110.

De Coster, M., \& Pichault, F. (1998). Traité de sociologie du travail. Bruxelles: De Boeck.

Develay, M. (1994). Peut-on former les enseignants ? Paris: ESF.

Ecole nationale de formation agronomique de Toulouse. (2009). Etre professeur en établissement d'enseignement agricole, from http://www.enfa.fr/fr/wpcontent/uploads/2009/05/referentiel-metier-enseignant-enfa2009.pdf

Fisher, C., Filby, N., Marliave, R., Cahen, I., Dishaw, M., Moore, J., \& Berliner, D. (1978). Teaching behaviors, academic learning lime, and student achievement : Final report of Phase IIl-B, Beginning Teacher Evaluation Study. San Fransisco: Far West Educational Laboratory for Educational Research and Development.

Goigoux, R. (2006). Ressources et contraintes dans l'enseignement de la lecture au cours préparatoire. In B. Schneuwly \& T. Thevenaz-Christen (Eds.), Analyses des objets enseignés ; le cas du français (pp. 67-91). Bruxelles: De Boeck.

Guin, D., \& Trouche, L. (1999). The Complex Process of Converting Tools into Mathematical Instruments. The Case of Calculators. The International Journal of Computers for Mathematical Learning, 3(3), 195-227.

Leblanc, S., Ria, L., Dieumegard, G., Serres, G., \& Durand, M. (2008). Concevoir des dispositifs de formation professionnelle des enseignants à partir de l'analyse de l'activité dans une approche enactive. @ctivités, 1, 58-78.

Lenoir, Y. (1993). Regard sur les rapports entre savoir et didactique, différents sens pour les didactiques. In P. Jonnaert \& Y. Lenoir (Eds.), Sens des didactiques et didactique du sens. Sherbrooke, Québec, Canada: Editions du CRP.

Lenoir, Y. (2010). La notion de référentialité dans la formation à l'enseignement. Recherche et formation, 64, 91-104.

Lenoir, Y., \& Bouillier-Oudot, M.-H. (2006). Savoirs professionnels et curriculum de formation. Québec, Canada: Presses de l'Université Laval.

Marcel, J.-F. (2004). Recherches contextualisées et pratiques enseignantes. In J.-F. Marcel \& P. Rayou (Eds.), Recherches contextualisées en éducation (pp. 13-26). Paris: INRP.

Marcel, J.-F. (2009). Le Sentiment d'Efficacité Professionnelle, un indicateur pour connaître le développement professionnel des "nouveaux" professeurs de l'enseignement agricole français. Questions Vives, 5(11), 161-176.

Marcel, J.-F., Murillo, A., \& Bouillier-Oudot, M.-H. (2011). Rapport d'étape $n^{\circ} 1$. Le travail des enseignants dans les établissements de l'enseignement agricole public. Stabilisation d'un dispositif d'analyse. Auzeville: Ecole Nationale de Formation Agronomique.

Maurice, J.-J. (2006). L'expérience de l'enseignant : une réflexivité limitée. Formation et pratiques d'enseignement en questions, 3, 53-67.

Mayen, P., Métral, J.-F., \& Tourmen, C. (2010). Les situations de travail, des références pour les référentiels. Recherche et formation, 64, 31-46.

Murillo, A. (2010). Le niveau de difficulté des tâches scolaires : des marges de manœuvre limitées pour les enseignants. Carrefours de l'Education, 29, 79-93.

Numa Bocage, L., Clauzard, P., \& Monchaux, P. (2011). Utilisation du TBI et transformations du travail enseignant Actes $d u$ colloque international INRP. Lyon: INRP.

Rey, B. (2010). Les référentiels. Recherche et formation, 64, 117-120. 
Rogalski, J. (2003). Y a-t-il un pilote dans la classe ? Une analyse de l'activité de l'enseignant comme gestion d'un environnement dynamique ouvert. Recherches en Didactique des Mathématiques, 23(3), 343-388.

Tanguy, L. (1994). Rationalisation pédagogique et légitimité politique. In F. Rope \& L. Tanguy (Eds.), Savoirs et compétences, de leur usage dans l'école et l'entreprise. Paris: L'Harmattan.

Tardif, M., \& Lessard, C. (1999). Le travail enseignant au quotidien. Laval: Presses de l'Université de Laval.

Trinquier, M.-P. (2010). Enseignement, représentations et pratiques. Confronter le sociocognitif au pragmatique: continuités et ruptures d'une relation. Note de synthèse pour l'Habilitation à Diriger des Recherches en sciences de l'éducation, Université de Toulouse, Toulouse.

Van Zanten, A. (2002). La profession enseignante en France : quelles évolutions ? Revue internationale d'éducation de Sèvres, 30, 85-93.

Veyrac, H., \& Bouillier-Oudot, M. H. (2011). Les concepts de représentations de la tâche en ergonomie pour la formation professionnelle des enseignants débutants. In P. Maubant \& S. Martineau (Eds.), Fondements des pratiques professionnelles des enseignants (pp. 219-242). Ottawa: Presses de l'Université d'Ottawa.

Veyrunes, P., Gal-Petitfaux, N., \& Durand, M. (2007). La lecture orale au cycle 2 : configuration et viabilité de l'activité collective dans la classe. Repères, 36, 59-76.

Wanlin, P., \& Crahay, M. (2009). Hétérogénéité des élèves et gestion de la classe : quels dilemmes et comment les surmonter ? Communication dans le symposium "Les pratiques d'enseignement et la prise en compte de l'hétérogénéité des élèves" coordonnée par L. Talbot Actes du colloque Recherche en Education et Formation. Nantes. 